\title{
First results from the RAPID imaging energetic particle spectrometer on board Cluster
}

\author{
B. Wilken ${ }^{1, *}$, P. W. Daly ${ }^{1}$, U. Mall ${ }^{1}$, K. Aarsnes ${ }^{2}$, D. N. Baker ${ }^{3}$, R. D. Belian ${ }^{4}$, J. B. Blake ${ }^{5}$, H. Borg ${ }^{6}$, J. Büchner ${ }^{1}$,

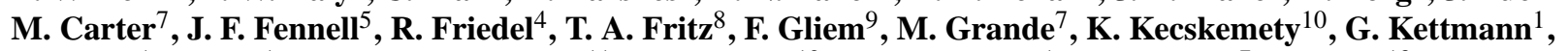 \\ A. Korth ${ }^{1}$, S. Livi ${ }^{1}$, S. McKenna-Lawlor ${ }^{11}$, K. Mursula ${ }^{12}$, B. Nikutowski ${ }^{1}$, C. H. Perry ${ }^{7}$, Z. Y. Pu ${ }^{13}$, J. Roeder ${ }^{5}$, \\ G. D. Reeves ${ }^{4}$, E. T. Sarris ${ }^{14}$, I. Sandahl ${ }^{15}$, F. Søraas ${ }^{2}$, J. Woch ${ }^{1}$, and Q.-G. Zong ${ }^{1}$ \\ ${ }^{1}$ Max-Planck-Institut für Aeronomie, Katlenburg-Lindau, D-37191 Katlenburg Lindau, Germany \\ ${ }^{2}$ University of Bergen, Allegt. 55, 5007 Bergen-U, Norway \\ ${ }^{3}$ LASP, Boulder-CO 80309, USA \\ ${ }^{4}$ LANL, Los Alamos-NM 87545, USA \\ ${ }^{5}$ Aerospace Corporation, Los Angeles-CA 90009, USA \\ ${ }^{6}$ IRF, 90187 Umea, Sweden \\ ${ }^{7}$ RAL, Chilton, Didcot, Oxfordshire OX110QX, UK \\ ${ }^{8}$ Boston University, Boston-MA 02215, USA \\ ${ }^{9}$ IDA, D-38106 Braunschweig, Germany \\ ${ }^{10}$ KFKI, H-1525 Budapest-114, Hungary \\ ${ }^{11}$ NUI, Maynooth-Co., Kildare, Ireland \\ ${ }^{12}$ University of Oulu, 90571 Oulu, Finland \\ ${ }^{13}$ Peking University, Beijing 100871, China \\ ${ }^{14}$ University of Thrace, Xanthi, Greece \\ ${ }^{15}$ IRF,S-98128 Kiruna-C, Sweden \\ *The RAPID team deeply regrets the untimely demise of B. Wilken, PI of the Cluster project. Without him, the RAPID \\ instrument would never have been created
}

Received: 9 May 2001 - Revised: 10 September 2001 - Accepted: 12 September 2001

\begin{abstract}
The advanced energetic particle spectrometer RAPID on board Cluster can provide a complete description of the relevant particle parameters velocity, $V$, and atomic mass, $A$, over an energy range from $30 \mathrm{keV}$ up to $1.5 \mathrm{MeV}$. We present the first measurements taken by RAPID during the commissioning and the early operating phases. The orbit on 14 January 2001, when Cluster was travelling from a perigee near dawn northward across the pole towards an apogee in the solar wind, is used to demonstrate the capabilities of RAPID in investigating a wide variety of particle populations. RAPID, with its unique capability of measuring the complete angular distribution of energetic particles, allows for the simultaneous measurements of local density gradients, as reflected in the anisotropies of $90^{\circ}$ particles and the remote sensing of changes in the distant field line topology, as manifested in the variations of loss cone properties. A detailed discussion of angle-angle plots shows considerable differences in the structure of the boundaries between the open and closed field lines on the nightside fraction of the pass and the magnetopause crossing. The 3 March 2001 encounter of Cluster with an FTE just outside the magnetosphere is used to show the first structural plasma investigations of an FTE by energetic multi-spacecraft observations.
\end{abstract}

Correspondence to: U. Mall (mall@linmpi.mpg.de)
Key words. Magnetospheric physics (energetic particles, trapped; magnetopause, cusp and boundary layers; magnetosheath)

\section{The instrument}

The RAPID spectrometer (Research with Adaptive Particle Imaging Detectors), described in detail by Wilken et al. (1995), is an advanced particle detector for the analysis of suprathermal plasma distributions in the energy range from $20-400 \mathrm{keV}$ for electrons, $30 \mathrm{keV}-1500 \mathrm{keV}$ for hydrogen, and $10 \mathrm{keV} /$ nucleon-1500 keV for heavier ions. Innovative detector concepts, in combination with pinhole acceptance, allow for the measurement of angular distributions over a range of $180^{\circ}$ in the polar angle for electrons and ions. Identification of the ion species is based on a two-dimensional analysis of the particle's velocity and energy. Electrons are identified by the well-known energy-range relationship. Table 1 list the main parameters of the RAPID instrument.

The energy signals in RAPID are analyzed in 8 bit ADCs. With a mapping process the 256 channels are reduced to 8 channels in the case of the ion sensor and into 9 channels in the case of the electron sensor. The resulting energy channel limits are listed in Table 2. 
Table 1. Specifications for the RAPID spectrometer

\begin{tabular}{|c|c|c|}
\hline Energy ranges: & $\begin{array}{l}\text { Hydrogen } \\
\text { Helium } \\
\text { CNO } \\
\text { Electrons }\end{array}$ & $\begin{array}{l}30-1500 \mathrm{keV} \\
100-1500 \\
105-1500 \\
20-400\end{array}$ \\
\hline $\begin{array}{l}\text { Mass range: } \\
\text { Resolution }(A / d A) \text { : } \\
\text { Field-of-view: }\end{array}$ & $\begin{array}{l}\text { Oxygen } \\
\text { IIMS (Ions) } \\
\text { IES (Electrons) }\end{array}$ & $\begin{array}{l}1,4,12-16,28-56 \mathrm{amu} \\
4 \\
\pm 3^{\circ} \times 180^{\circ} \\
\pm 17.5^{\circ} \times 180^{\circ}\end{array}$ \\
\hline $\begin{array}{l}\text { Geometry factor: } \\
\text { (for } 180^{\circ} \text { ) }\end{array}$ & $\begin{array}{l}\text { IIMS } \\
\text { IES }\end{array}$ & $\begin{array}{l}2.6 \times 10^{-2} \mathrm{~cm}^{2} \cdot \mathrm{sr} \\
2.0 \times 10^{-2}\end{array}$ \\
\hline
\end{tabular}

Ions and electrons are detected by two independent subinstruments, the IIMS (Imaging Ion Mass Spectrometer) for ions and the IES (Imaging Electron Spectrometer) for electrons. The IIMS itself consists of three sensor heads (see Fig. 1). Each sensor head subdivides the original $60^{\circ}$ acceptance angle into 4 segments of $15^{\circ}$ each, so that the IIMS covering the $180^{\circ}$ in the plane containing the spacecraft spin axis can sort the incoming particles into 12 directional bins. In the other direction, that of the spacecraft's rotation, the particles are highly collimated to within a field-of-view of $6^{\circ}$. The data are collected in 16 spin sectors during one rotation, synchronised by the Sun pulse which places the Sun in sector 13. Note that the spacecraft spin axis is directed towards the ecliptic south pole, which means as it is viewed from the north, the spacecraft rotates clockwise. In summary, the ion spectral counts are sorted into 8 energy bins, 12 polar angle segments, 16 azimuthal sectors, and 3 mass ranges. Higher energy and mass precision is obtained with so-called 'direct events'.

The IES system, like the IIMS, is arranged in three units, each is covering $60^{\circ}$ in polar direction, and each is subdivided into $20^{\circ}$ 'pixels'. Thus, there are 9 microstrip solidstate detectors, one for each polar segment. The electron spectral counts are also sorted into 16 azimuthal sectors, but only 9 polar segments.

\section{Assessment of the RAPID commissioning}

The Cluster Commissioning phase started in August and lasted until the end of November 2000. During this time, each instrument was turned on and tested, one at a time on each spacecraft, without any of the other instruments operating.

An important factor for RAPID is that the sensor is an open design which is sensitive for solar UV-photons. During each spin, the instrument faces the Sun for approximately $70 \mathrm{~ms}$. Out of the 3 sensor systems (compare Fig. 1), the center system S2 is primarily illuminated. The photons falling into the active area of the sensor release photoelectrons from the entrance foil (Al coated Lexan foil) or they are transmit-
Table 2. RAPID Energy Channel definitions (approximate values)

\begin{tabular}{cllll}
\hline Channel & \multicolumn{1}{c}{$\mathrm{H}$} & \multicolumn{1}{c}{$\mathrm{He}$} & \multicolumn{1}{c}{$\mathrm{CNO}$} & electrons \\
\hline 1 & $30 \mathrm{keV}$ & $100 \mathrm{keV}$ & $105 \mathrm{keV}$ & $20 \mathrm{keV}$ \\
2 & 68 & 140 & 300 & 46 \\
3 & 95 & 175 & 460 & 64 \\
4 & 170 & 240 & 560 & 90 \\
5 & 410 & 365 & 730 & 123 \\
6 & $1.1 \mathrm{MeV}$ & 785 & $1.1 \mathrm{MeV}$ & 172 \\
7 & 3.1 & $1.9 \mathrm{MeV}$ & 1.7 & 240 \\
8 & 27. & 4.4 & 3.6 & 330 \\
upper limit & 200. & 20. & 9.0 & 400 \\
\hline
\end{tabular}

ted into the interior of the TOF-system and then scattered into the channel plates. In both cases, the channel plates create an unrelated start and/or stop signal. According to investgations in the laboratory, we selected the foil thickness and its Al coating such that the UV-flux should be reduced to a tolarable level. During the commissioning phase, we noticed light induced counting rates which were higher than the predicted levels. Our first investigations indicate that the gain of the channel plates of S2 most likely shows a decrease in sensitivity. From the sophisticated in-flightcalibration facility, we can exclude any deterioration of the electronic system. The described effect can be best noticed when we plot the count rate as an angle-angle plot of the polar angle versus the azimuthal angle (see Fig. 2).

As can be seen from the data taken during 14 January 2001 , the count rates are measured in all sectors, while in the frame taken during 3 March 2001, the center strip in the polar angular interval indicates a loss of sensitivity. Tests currently in progress indicate that by increasing the channel plate, the bias voltage reduces the loss of sensitivity in S2.

A second, more subtle problem surfaced during the commissioning phase which had to do with the applied priority scheme in the DPU of the instrument: as mentioned previously, the DPU returns a small fraction of data as direct events (DE) with the highest resolution in energy, $E$, time, $T$, and direction DIR. To favour heavy particles, a priority scheme was invented. A direct check of the DEs during the commisioning phase revealed that the DPU had loaded a scheme with an even weight factor for all ion species. This means that the returned data reflect the natural distribution of the measured particles. A software fix was uploaded on 14 April 2001 which improved the statistical identification of heavy particles.

\section{Dawn-dusk meridian tail crossings}

Off-equator correlated multi-satellite observations at the magnetopause was the main objective of the ISEE $1 / 2$ pair, launched in 1977. The two spacecraft of the ISEE mission were flying in a similar stretched elliptical orbit (apogee/perigee: 22/ $5 R_{E}, i=20^{\circ}$ ) around the Earth and 

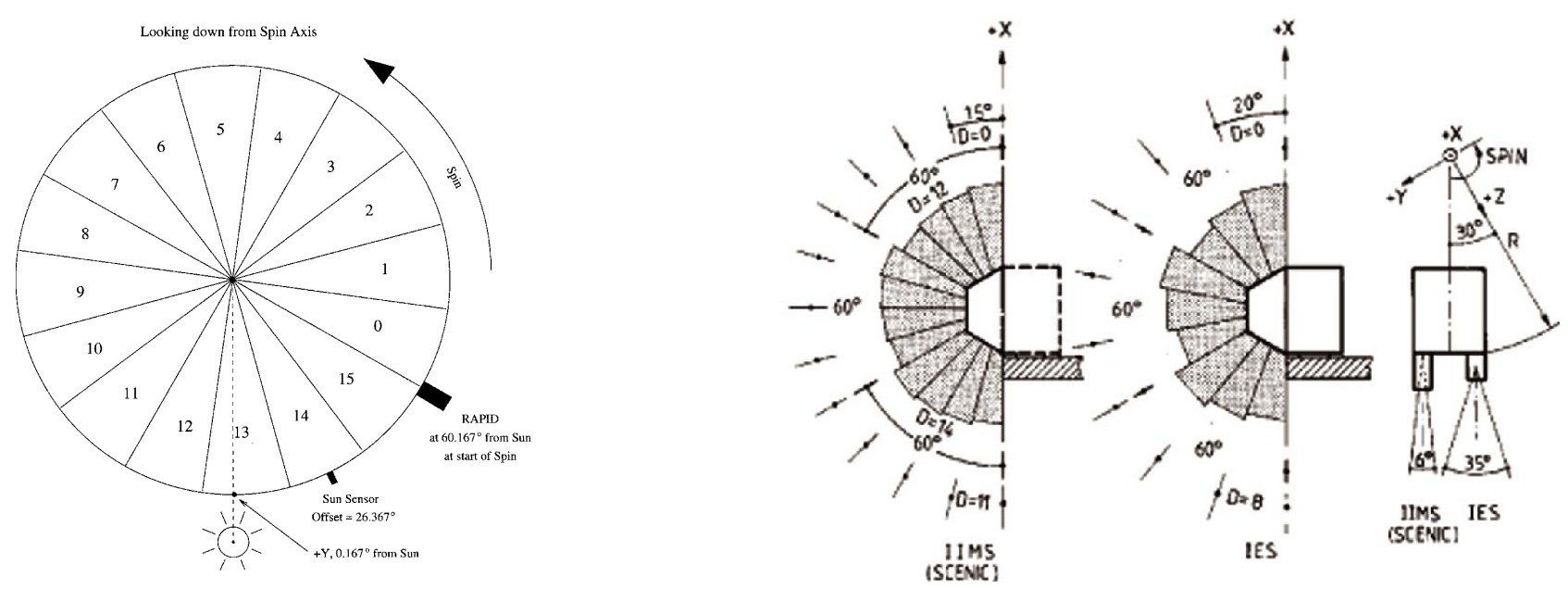

Fig. 1. Azimuthal (left figure) and polar (right figure) sectoring scheme of RAPID.

similar to Cluster, ISEE was crossing the dayside magnetopause in a nearly orthogonal fashion.

Long before the launch of Cluster, the dual satellite ISEE returned a wealth of important measurements about a moving magnetopause, about reconnection sites at the dayside magnetopause, called FTEs, and about magnetospheric responses to changes in the solar wind, processes today denoted as SunEarth relations. A more recent important single-spacecraft mission to the frontside magnetosphere with extensive magnetopause skimming intervals was the Japanese GEOTAIL in its second orbital phase since November 1994. Its elliptical Earth trajectory in the ecliptic plane provided two times a year exceptionally good matches to the dayside shape of the magnetopause, thus allowing for hours of observations in or near the magnetopause current sheet. As mentioned before, Cluster magnetopause crossings at the frontside are orthogonal in nature; however, the three-dimensional satellite constellation should capture kinematic magnetopause properties under all circumstances.

One of the outstanding features of RAPID is the complete coverage of the unit sphere in velocity space with 192 continous pixels. A similar coverage was achieved with the energetic particle instrument on ISSE-1. However, the ISSE-1 instrument was a single sensor head instrument mounted on a stepping platform with no mass resolution. The polar angle, was stepped once per spin. This means that a complete scan of the polar angle which takes only $4 \mathrm{~s}$ for Rapid including mass identification, can now be obtained in a fraction of what one could do in the past. The purpose of the following section is meant to show these cababilities in sequences of angle-angle plots for selected intervals of the orbit on 14 January. It should be noted that the inter-satellite separation was of the order of $600 \mathrm{~km}$ which is, in fact, close to the gyroradii of $50 \mathrm{keV}$ protons in the region of interest. Inspection of the data from satellites 1, 3 and 4 revealed few differences in energetic particle distribution. For this reason, we restrict our discussion to a single spacecraft.
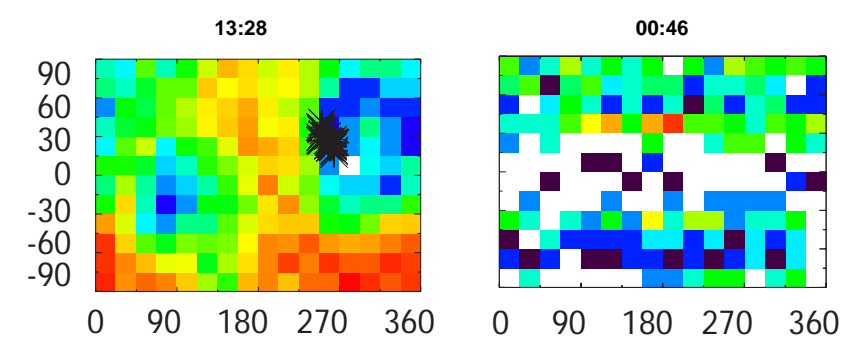

Fig. 2. $\mathrm{H}^{+}$fluxes shown as an angle-angle plot for 14 January 2001 and 3 March 2001.

\subsection{Observations on 14 January 2001}

As shown in Fig. 3 on 14 January 2001, the four Cluster satellites travelled on a trajectory close to the dawn-dusk meridian with an inter-satellite separation of approximately $600 \mathrm{~km}$. During the perigee pass, the RAPID instruments were in an OFF state. Shortly after ground contact was established, Cluster-3 (CL-3), which was selected as a reference for this paper, found itself in a region of space populated by trapped energetic particles, as indicated in the initial phase of the spin-averaged polar and azimuth distributions in the central panels of Fig. 4. The bottom panel indicates that the RAPID spectrometer on spacecraft 3 was activated at 04:15 UT and remained ON until the end of the day. The upper three panels of Fig. 4 show spectrograms for the three selected species of hydrogen, helium, and oxygen (or short CNO group; RAPID is not designed to resolve the three constituents of this group).

In a global sense, Fig. 4 shows the Cluster assembly in a pass from perigee at dawn, across the North Pole, through the dusk magnetosphere, to an exit into the magnetosheath at about 16:00 LT. This trajectory took the four spacecraft from an initial region characterized by the presence of trapped particles (located about 04:15 to 04:45 UT), as indicated by the azimuthal and polar distributions of proton count rates 


\section{Cluster spacecraft position on 14.01.2001}
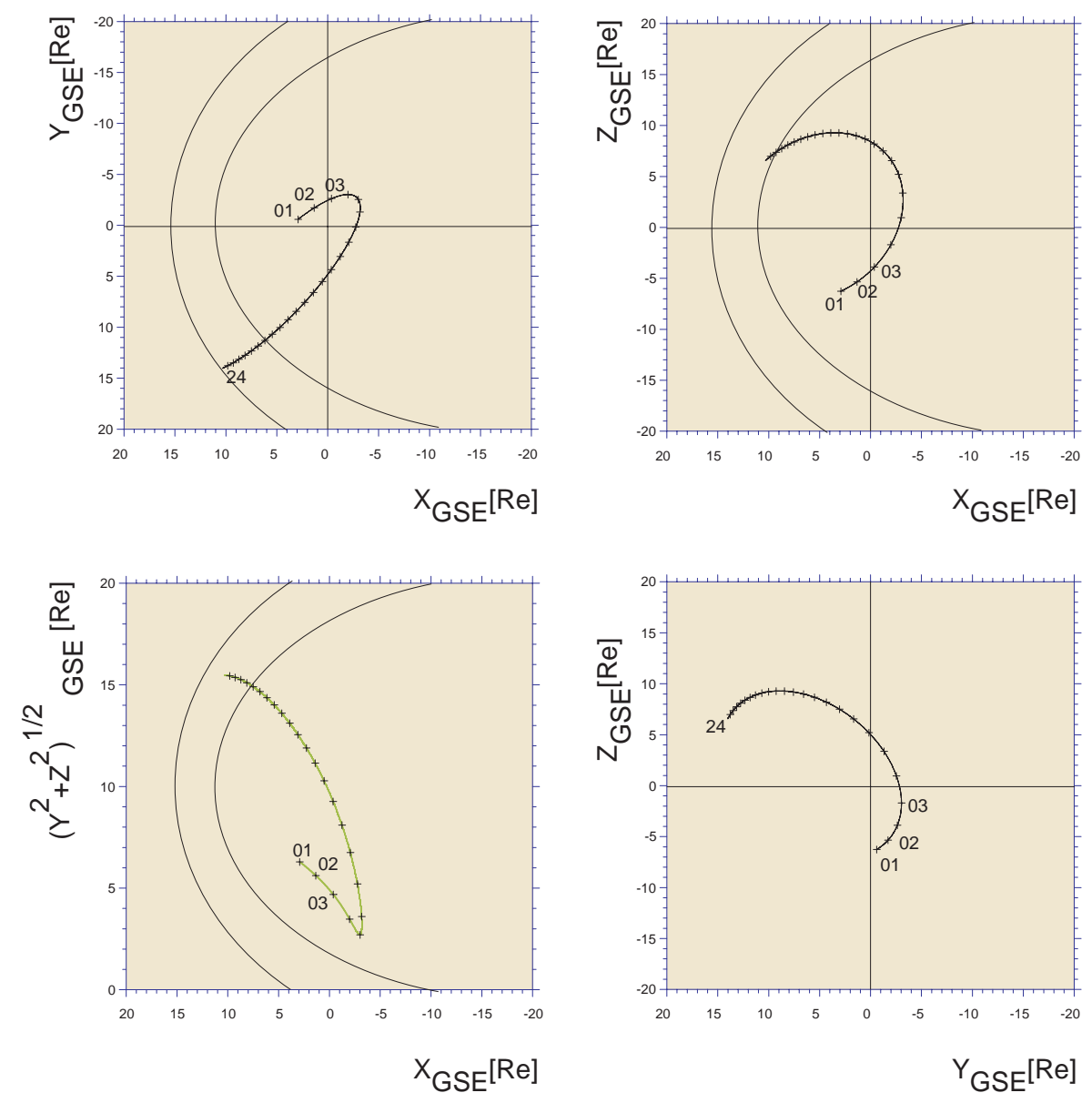

Fig. 3. The orbit of the Cluster fleet on 14 January 2001. The tick marks denote the spacecraft position in intervals of one hour.

in Fig. 4. After 04:45 UT, Cluster entered a region of space which was essentially void of energetic particles until 10:50 UT. Inspection of the orbit plot in Fig. 3 suggests that this region corresponds to the polar cap which is expected to be, by and large, without an energetic particle population because the field lines are open to the solar wind.

At 10:45 UT spacecraft 2 re-entered a region with isotropic particle distributions (Fig. 4) and at 15:00 UT, the satellites penetrated the high-latitude magnetopause on the outbound leg of the trajectory. Close inspection of Fig. 4 reveals an indication for an interaction with the bow shock or with field lines connecting to the bow shock in the last two hours of the day.

The basic magnetospheric features visited by Cluster throughout this tour on 14 January 2001 are schematically depicted in Fig. 5, as seen from a tail position.

It appears that Cluster at 04:15 UT barely scratched the northern edge of the nightside region with closed field lines, which corresponds to the northern edge of the plasma sheet; by 04:45 UT, Cluster has reached open field lines emanating from the polar cap.

\subsection{Entry into the trapped region}

Figures 6 and 7 show the complete time series of angleangle plots measured by the three RAPID instruments on spacecraft 1, 3 and 4 for the time period between 10:30 and 15:48 UT on 14 January 2001. Spacecraft 2 was turned off during this time period.

Each frame in Figs. 6 and 7 shows the $H$ flux in the energy range between 28 and $68 \mathrm{keV}$, measured in 12 polar directions and 16 azimuthal sectors as a mercator projection of the spherical coordinates (Polar angle and azimuthal angle). The instrument accumulates the ions in telemetry normal mode over 32 spins so that we can produce one frame each $32 \times 4 \mathrm{~s}=128 \mathrm{~s}$. In telemetry burst mode the accumulation period is $8 \times 4 \mathrm{~s}=32 \mathrm{~s}$.

In the individual angle-angle plots, the data were shifted so that the Sun sector, which is in sector 13 , is displayed at $360^{\circ}$. At this point, it should be noted that charged particles are guided and ordered by the magnetic fields in space and as a result, the angular distributions can reflect the direction of the magnetic field vector. The particles which move close to the magnetic field line will show a loss cone effect, 


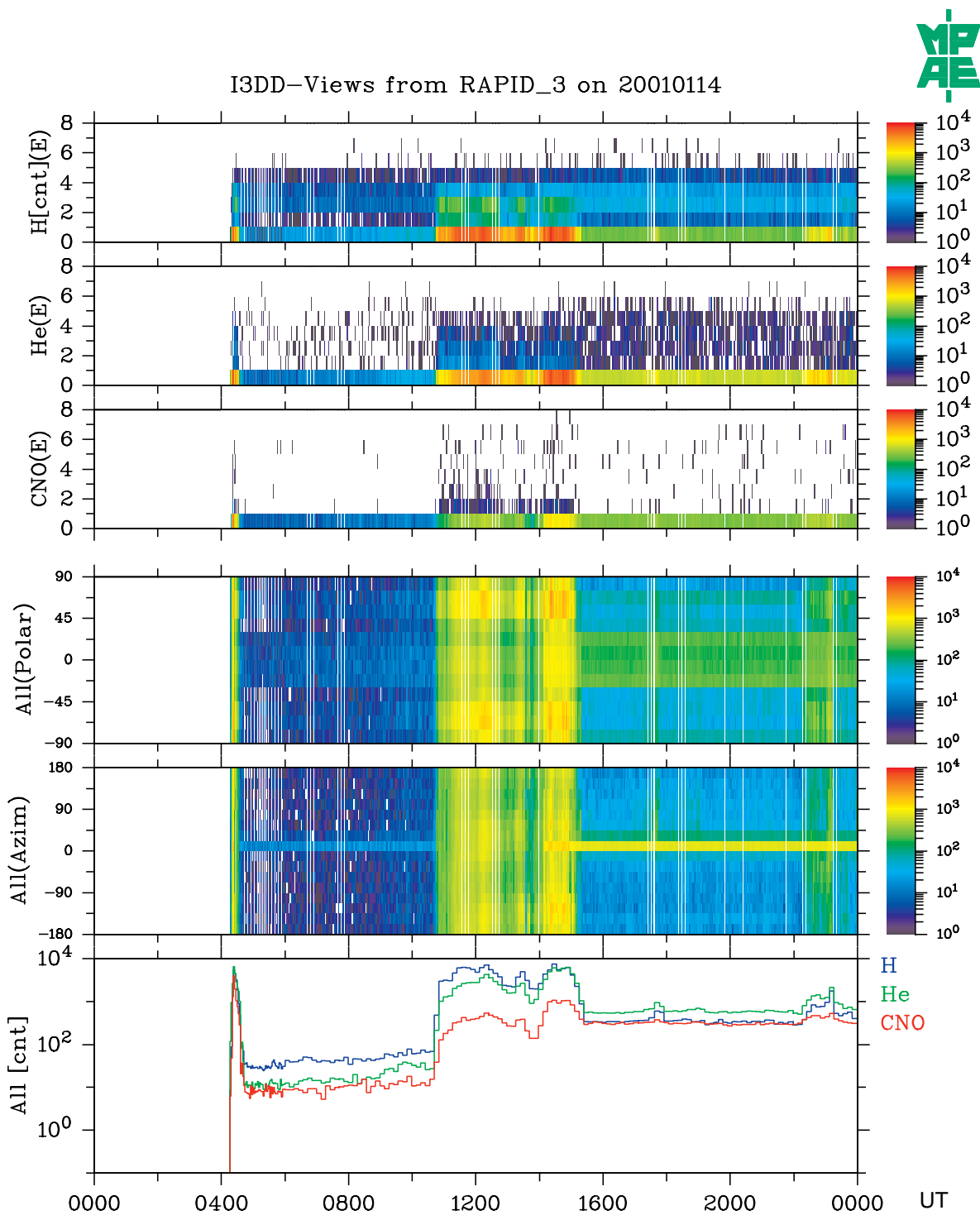

Fig. 4. Count rates measured by RAPID on spacecraft 3 on 14 January 2001. The panels show from top to bottom the H, He CNO count rates, the polar distribution of the total count rate, the azimuthal total count rate and the total accumlated count rate.

i.e. these particles will reach atmospheric altitudes and will be scattered. These loss cones can be directly observed by RAPID.

The time series in Fig. 6 shows the transition from the low flux polar cap/lobe fields (the time window of 10:31 UT to about 10:42 UT) into a region filled with energetic ions. The initial angular distributions, e.g. 10:44 to 11:24 UT and beyond, are irregular for some time.

When RAPID enters from a region of open field lines into a region of closed field lines (trapped population), we expect to observe first the particles which have the largest gyro-radii. These are the particles which have $90^{\circ}$ pitchangles and have, per definition, no velocity component along the field lines. For this reason, a loss cone cannot be observed. However, when the spacecraft starts to penetrate deep enough into the trapped region, they can also observe the par- ticles with a smaller pitch-angle.

The initial approach phase in Fig. 6 (frames 10:37 to 11:07 UT) is in reasonable agreement with the picture of a well-defined boundary between different particle populations (here, between open and closed field lines) which has a thickness of two gyro-radii. According to the orbit in Fig. 3, this boundary crossing occurred near 18:00 LT at high-latitudes $\left(Z=9 R_{E}\right)$. This translates into a prevailing direction of the local magnetic field toward Earth (schematically shown in Fig. 3). The earliest indication for the approach of particle loaded field lines can perhaps be seen in the frame labelled 10:45 UT. The frames 10:40 and 10:41 UT are most likely distorted by aliasing since the boundary speed was too high for the effective RAPID time resolution. The frames 10:48, 10:52, 10:54, and 11:09 UT are examples of situations with nearly equal north-south intensities. The space- 


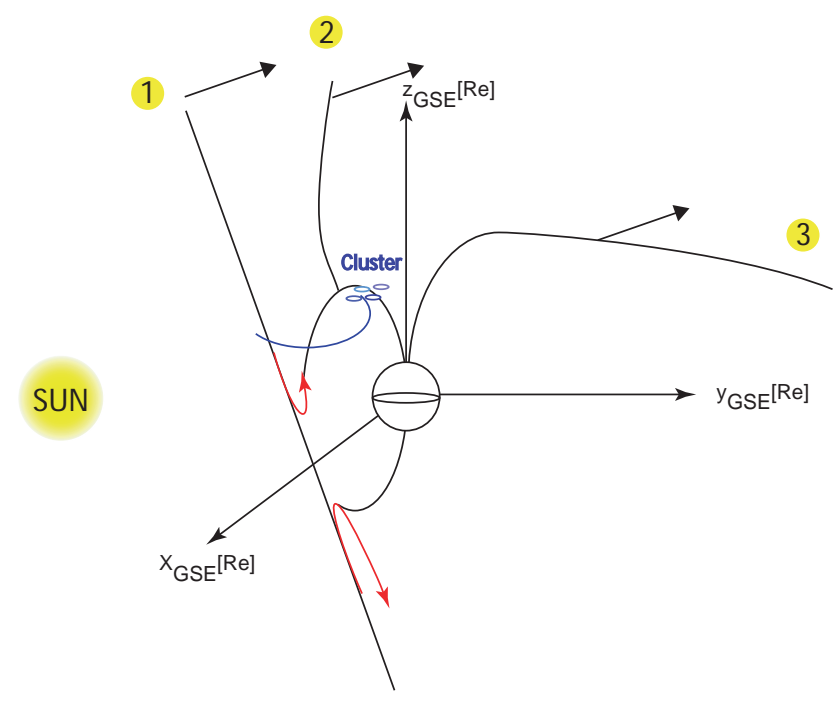

Fig. 5. Schematics of the time sequence of reconnected field lines at the dayside magnetopause. The Cluster path on 14 January 2001 is indicated by a blue line.

craft must have penetrated the layer for a distance exceeding $d=2 r$, with $r$ denoting the particle's gyro-radius. The frame 11:00 UT shows only a populated south side, supporting the view that spacecraft 1 is rather close to the outer edge of the layer where the particles seem to come from one direction (south to north) and form a current sheet due to the strong density gradient. After 11:09 UT, the angular distributions become increasingly more symmetric in the north-south direction, implying a deep penetration of into the high intensity region.

3.3 Crossing the trapped region and penetrating the magnetopause

The frames between 11:23 and 15:30 UT display the entire pass through the trapped region from polar cap/lobe field lines at 10:31 UT to the exit into the magnetosheath near 15:11 UT. It is particularly interesting to note the slow evolution of the loss cones in the angle-angle distributions. The centers of these loss cones mark the entry and exit of the magnetic field vector. A particle population on a closed field line oscillates between the north and south mirror points, $\boldsymbol{B}_{m}$, in which all particle energy is converted to the perpendicular mode, i.e. the pitch-angle is $90^{\circ}$. The further away from the mirror points the particle observation is made, the smaller the width of the loss cone, which assumes a minimum at the point of minimum field strength along the field line (usually at the equator). For RAPID, the pixel size is $15^{\circ} \times 22.5^{\circ}$ so that any loss cone effect has to be comparable or larger than this field in order to be noticeable. The loss cones in Fig. 6 increase slowly from 11:09 UT (the first frames are part of the initial approach phase of the trapped region dominated by density gradient effects, as discussed in Sect. 3.2).

As one notices (e.g. RAPID 3) in Fig. 6, the angular width of the loss cones starts with small values at 11:09 UT and grows continually until 13:22 UT and for most part, the loss cones are pretty equal except for subtle differences over short periods of time. It is interesting and surprising to note that from around 13:13 UT onward, the magnetic field direction is no longer aligned with our loss cone structure. The loss cones grow faster than before and broaden up to $180^{\circ}$ around 13:27 UT. It should be noted here that the low energy electron detector PEACE on Cluster reported a rather short (1 min) detection of the cusp around 11:23 UT. The PEACE team argues that a change in solar wind conditions displaced the cusp sufficiently to bring it into the field-of-view of this instrument. Inspection of the respective frames at 11:22 and 11:24 UT in Fig. 7 gives no observational evidence for cusp effects in the RAPID energy range for ions.

It is interesting to note that from around 13:29 UT onward, one observes only $90^{\circ}$ pitch-angle particles, while the loss cone structures disappear. At the same time, one notices an increase in the total magnetic field (not shown) and observes a fluctuating $\boldsymbol{B}_{X}$-Geocentric Solar Ecliptic (GSE) magnetic field component which lasts for approximately $20 \mathrm{~min}$ while $\boldsymbol{B}_{Z}$ is positive. From the CIS quick-look data-plots, one can infer that a plasma with a more solar wind-like energy distribution is present during the same time interval, while the magnetic field shows the abrupt field variations.

The magnetic field signature is consistent with an FTE which was initiated at low-latitudes or with an early contact with the magnetopause. The evidence in the RAPID data which speaks for a flux transfer interpretation is the sudden disappearance of the loss cone structure, while the $90^{\circ}$ pitchangle particles remain observable. The disappearance in the loss cone distribution (frame 13:29 UT) is indicative of a change in the field topology due to the FTE being well below the Cluster point of observation. A magnetopause contact is unlikely since it would primarily affect the $90^{\circ}$ particles due to the large gyro-radii and it would not greatly affect the particles with small pitch-angles. We argue that the RAPID data favour an FTE interpretation.

While we are moving towards the magnetopause, whose crossing we identify by inspection of the magnetic field data and the disappearance of energetic particles in frame 15:16 UT (Fig. 7), we note that in frame 14:54 UT, the $90^{\circ}$ flux starts to be strongly reduced and the flux in one of the loss cones reaches a background count level. We interpret this as the immediate result of field line reconnection near the equator which interupts the streaming of particles along the field line from the southern mirror point. Small pitch-angles at the reconnection site move along the field until they are observed by RAPID as nearly $90^{\circ}$ particles. Although the data of the different RAPID instruments are not yet crosscalibrated, we can distinguish several features in the 3 measurement sequences. Close inspection of the data frames of the three spacecraft 1, 3 and 4 shown in Fig. 7 from 14:50 UT, 14:49 UT and 14:49 UT onward reveals significant structural differences in the plots. One can see that the loss cone structure is much better developed in the data recorded by spacecraft 3 and 4 than by spacecraft 1 .

We argue that spacecraft 3 and 4 observe particle distri- 

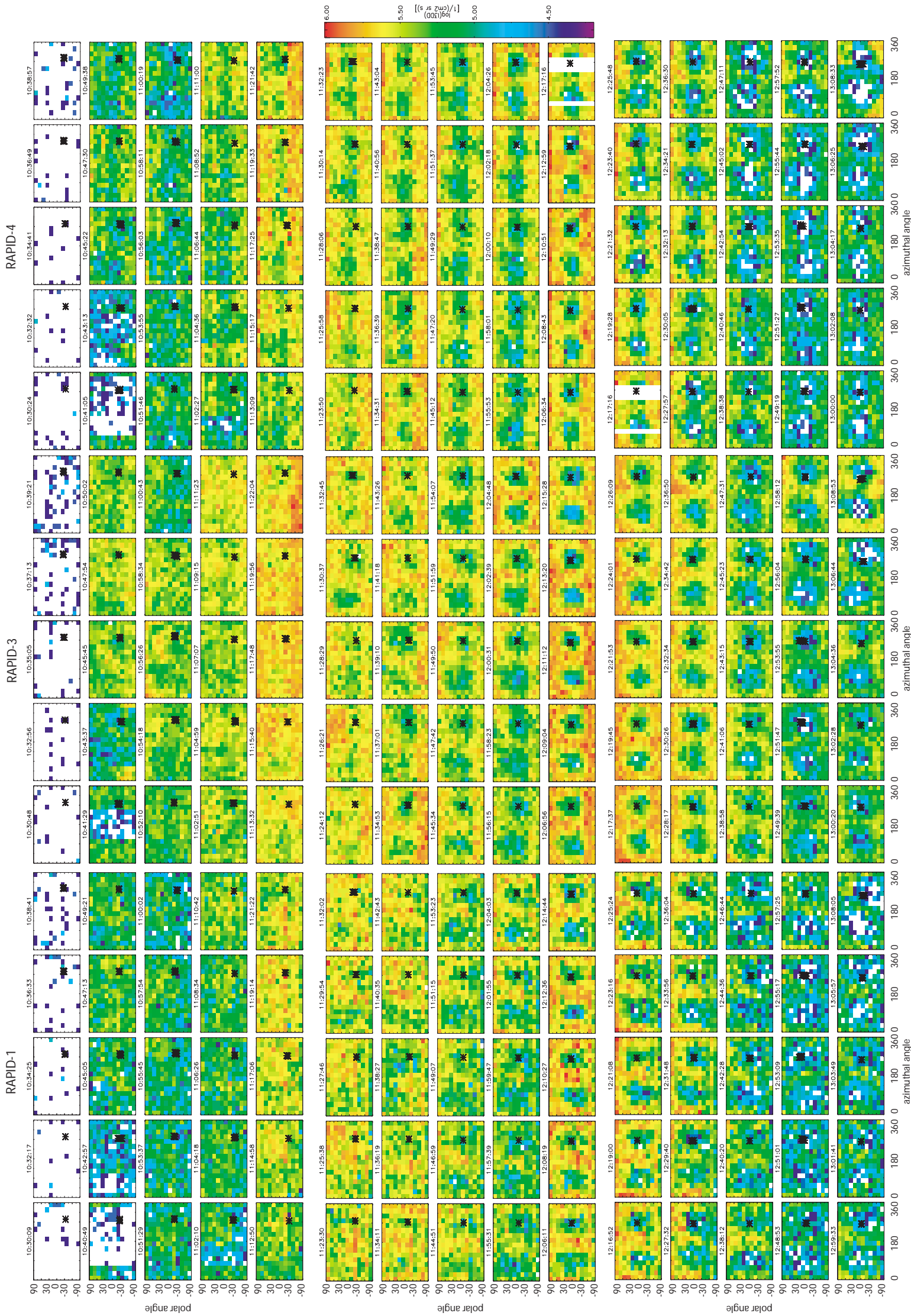

Fig. 6. Polar-azimuthal plots of $H$ flux measured by RAPID on spacecraft 1, 3, 4 on 14 January 2001 between 10:30 and 13:08 UT. 

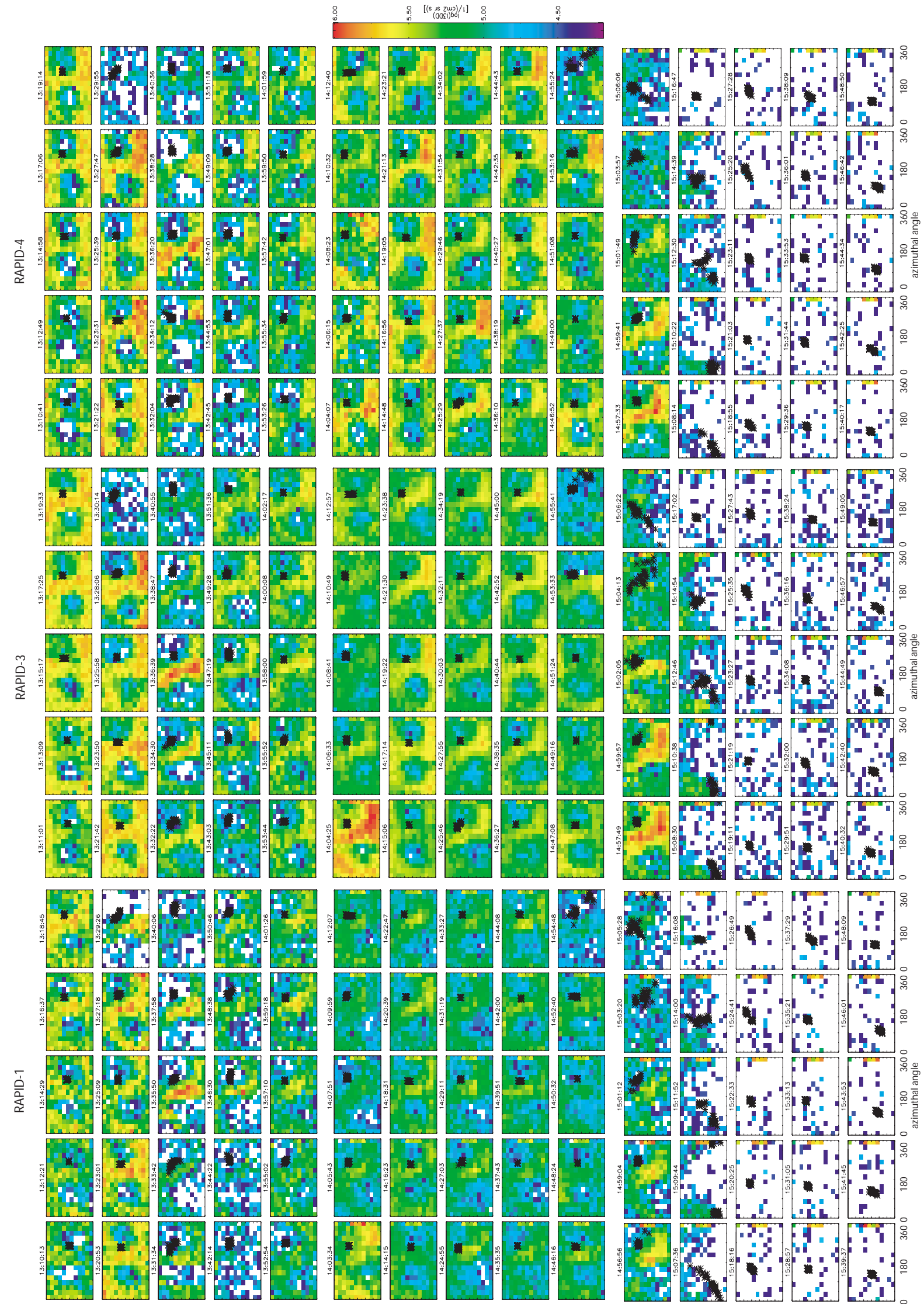

৪ 잉 \& \& 이유 \& 8 잉 \&

әбБиe sejod

ग|биe sejod

Fig. 7. Polar-azimuthal plots of $H$ flux measured by RAPID on spacecraft 1, 3, 4 on 14 January 2001 between 13:10 and 15:48 UT. 


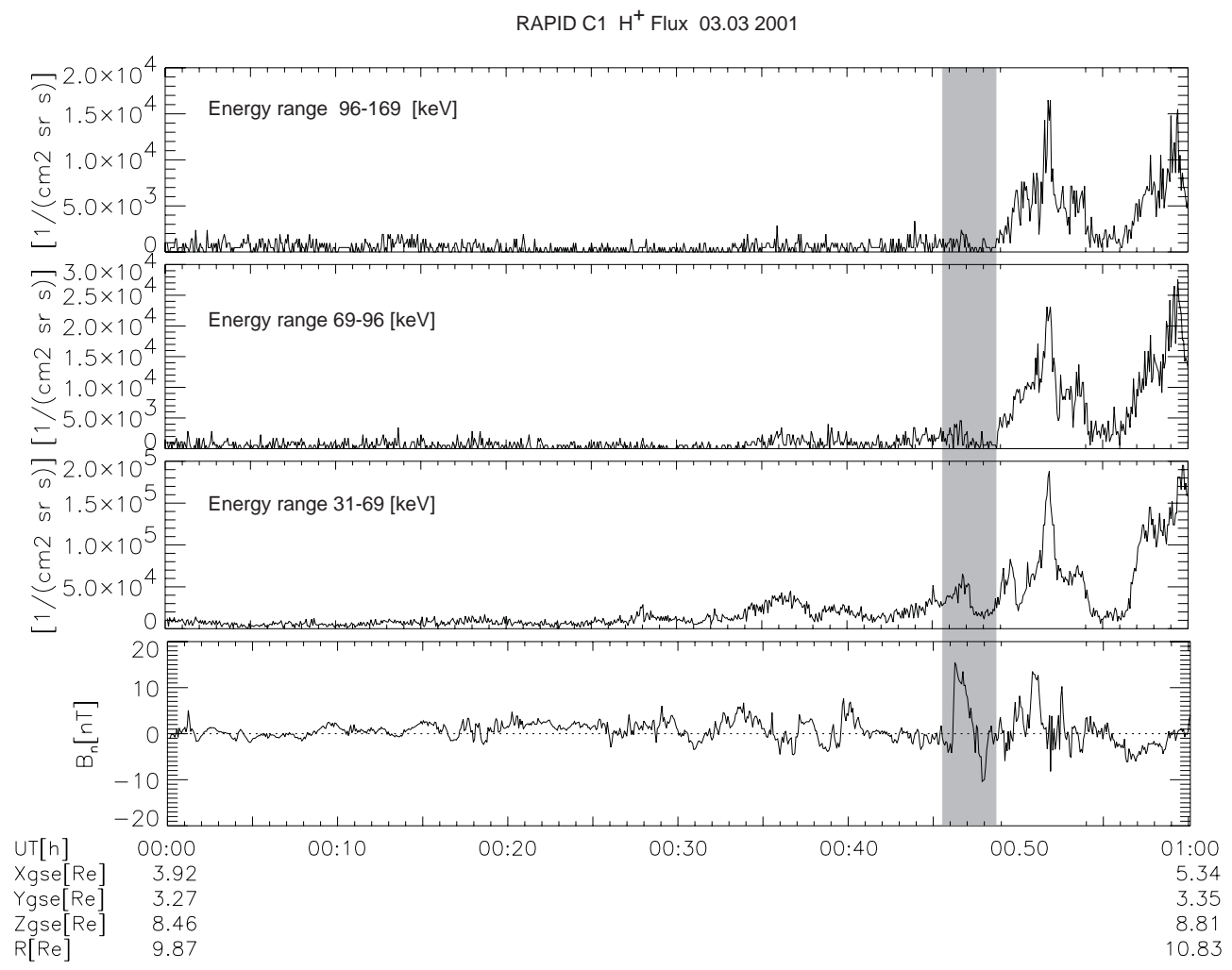

Fig. 8. Time development of the omnidirectional fluxes of the first three energy channels of RAPID and the magnetic field component normal to the magnetopause plane at spacecraft 1 .

butions on well closed field lines, while spacecraft 1 seems to observe the recently opened field lines. The sequence of frames starts to reveal, for the first time, the threedimensional signatures of an FTE in velocity space. It should be noted that ISEE-1 in the 1970s was able to observe similar angle-angle plots as RAPID does, today however, with a single sensor head mounted on a scanning platform. This meant that the timing resolution was closely tied to the spin motion. The detection technique did not allow for mass identification at that time. The novel approach of RAPID, which allows for a complete coverage of $4 \pi$ in velocity space, is responsible for the fact that we can now start exploring the spacial aspects of reconnection physics at the dayside. Figure 9 is a schematic of the above FTE event and the associated particle signature. A field line on the magnetopause is supposed to be opened in the vicinity of the equator which is about $10 R_{E}$ below the Cluster position. After the reconnection, the northern portion of the field line is swept across Cluster towards the tail (compare sequences 1, 2 and 3 in Fig. 9).

\section{High-latitude, dayside flux transfer event}

Another goal of Cluster is the study of the penetration of solar wind plasma and energy into the magnetosphere (Escoubet et al., 1997). Central issues to be investigated are reconnection processes, which can be large-scale, quasi-stationary, patchy or transient. It is thought to be the origin of 'Flux
Transfer Events' (FTEs) (Russell and Elphic, 1978). The reconnected flux tubes link the solar wind and magnetosphere. They allow solar wind plasma to enter the magnetosphere and magnetospheric plasma to escape (Scholer et al., 1981). One significant signature of FTEs are bipolar variations of the magnetic field component perpendicular to the magnetopause surface with $a+/-($ or $-/+)$ asymmetry in the northern (southern) hemisphere (Russell, 1994). The term FTE has become a generally accepted name for these observational signatures regardless of whether a proof of the magnetic connection between magnetosheath and magnetosphere exists (Papamastorakis, 1989). It was difficult to decide from single spacecraft observations whether one observed magnetic reconnection or the consequences of pressure pulses. Cluster offers new possibilities.

While the equatorial magnetopause region is thought to be the source region of FTEs (Daly et al., 1984), the highlatitude magnetopause is the natural place to look for their evolution. While the earlier observations, primarily by the ISEE spacecraft, were bound to low-latitudes (Daly et al., 1984), Cluster is in an ideal position to investigate these events at high latitudes. The Cluster fleet allows for the exploration of spatio-temporal structures of FTEs. With four spacecraft close by, taking measurements simultaneously, it becomes possible to investigate the structure and dynamics of FTE signatures. We report here the first multi-spacecraft energetic particle observations of an FTE-event observed at 


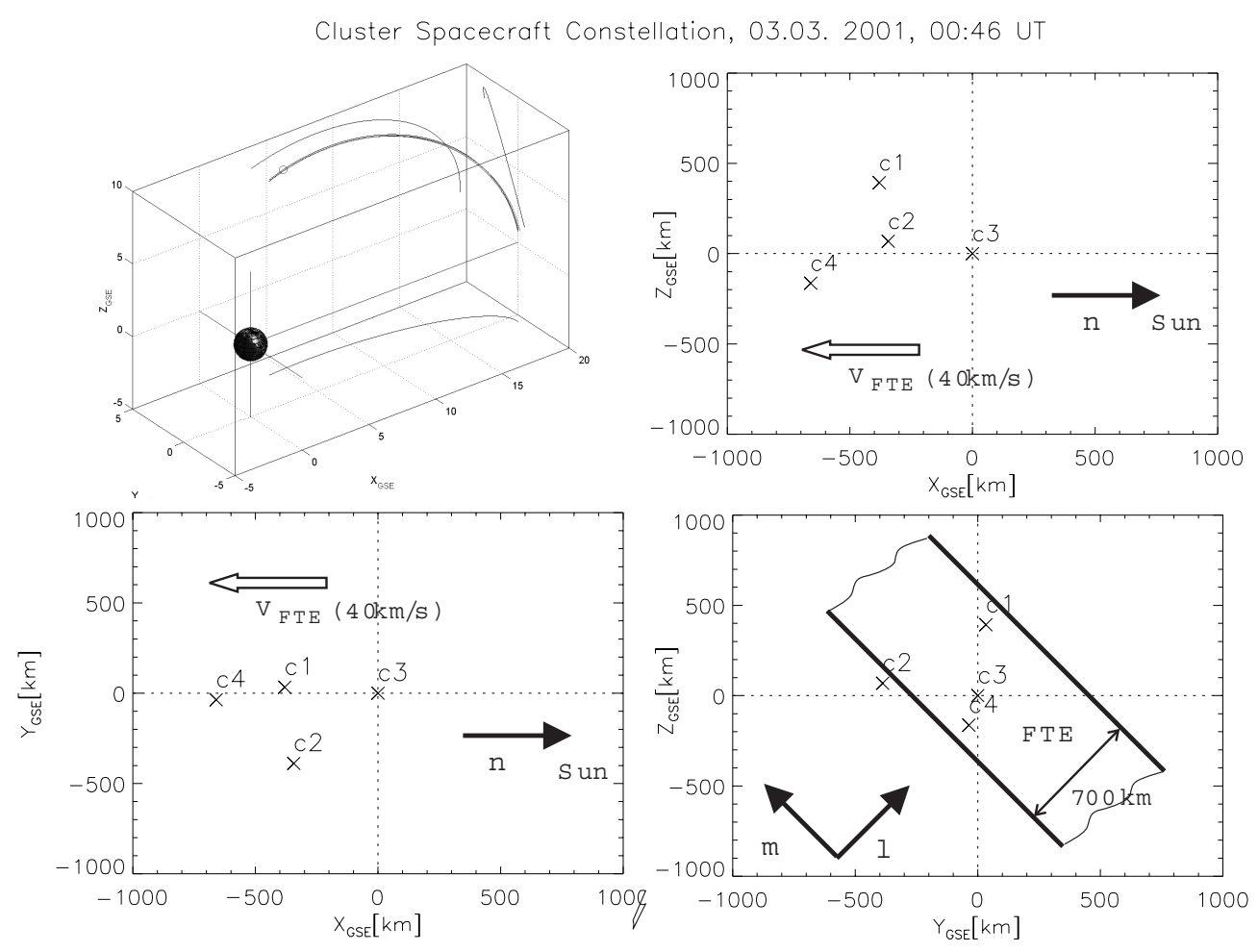

Fig. 9. The geometrical configuration for the four Cluster spacecraft on 3 March 2001 (upper left panel). Spacecraft positions relative to spacecraft 3 in GSE-coordinates at 00:46 UT (beginning of the first grey shaded internal of Fig. 8). The boundary normal coordinate system $(l, m, n)$ is marked by the solid arrows, the velocity component of the FTE motion $V_{\text {FTE }}$ is marked by an open arrow. Furthermore, a schematic plot of the FTE in the $Y-Z$-plane is given.

high-latitudes between 00:46 UT and 00:49 UT on 3 March 2001. Before this time interval, the $B_{Z}$-component of the interplanetary magnetic field has already been negative for four hours. Hence, reconnection at the dayside magnetopause is very probable.

\subsection{Observations on 3 March 2001}

The observations reported hereafter were made when Cluster had just left the magnetosphere at high-latitudes $\left(X_{\mathrm{GSE}}=\right.$ $5.3 R_{E}, Y_{\mathrm{GSE}}=3.3 R_{E}$, and $Z_{\mathrm{GSE}}=8.8 R_{E}$ ) and entered the magnetosheath near the outer cusp region at about 00:20 UT, according to CIS plasma diagnostics (CIS quicklook). Figure 9 shows the geometrical configuration during the period of observation. The Cluster orbit on 3 March is displayed in the upper left panel of Fig. 9, together with the projections of the 4 spacecraft trajectories. The circle on the trajectory designates the location where the FTE was encountered.

Figure 8 shows the omnidirectional proton fluxes in three energy channels HSPCT1 (30-68 keV), HSPCT2 (68$95 \mathrm{keV})$, HSPCT3 (95-170 keV), as measured by the RAPID instrument on spacecraft 1. Below the proton flux time series, the magnetic field component normal to the magnetopause plane is plotted. This was determined by the minimum variance analysis of magnetic field data (Sonnerup and Cahill, 1967) taken during the first hour of 3 March 2001.
An examination of the data measured on all 4 spacecraft reveals that starting at 00:30 UT, all three energy channels recorded a proton flux increase. Timely enhancements took place and they were accompanied by bipolar $B_{n}$ variations; therefore, they can be interpreted as FTEs. We will focus on the grey shaded time interval of 00:46-00:49 UT, when a bipolar variation of the normal magnetic field component $B_{n}$ indicates the encounter of an FTE. During the same time, all three energy channels show proton flux enhancements. We draw in Fig. 9 all spacecraft positions relative to spacecraft 3 at 00:46 UT and mark the unit vectors of the boundary normal coordinate system $(\boldsymbol{l}, \boldsymbol{m}, \boldsymbol{n})$ (Russell and Elphic, 1978). The normal $\boldsymbol{n}$ is directed in the $X_{\mathrm{GSE}}$ direction, and $\boldsymbol{l}$ and $\boldsymbol{m}$ are lying in the $X_{\mathrm{GSE}}-Y_{\mathrm{GSE}}$ plane. The structure is first observed by spacecraft 3 , then by spacecraft 1 and finally by spacecraft 4 . The delays indicate that the structure moves in the anti-sunward direction at a velocity of about $40 \mathrm{~km} / \mathrm{s}$ (deduced from the magnetic field observations). At the same time, CIS (see quicklook) observed a velocity of the surrounding bulk plasma of about $80 \mathrm{~km} / \mathrm{s}$. Hence, the FTE moves slower than the solar wind plasma, which is understandable due to the anchoring of the common flux tube in the magnetosphere.

Figure 10 displays the azimuthal-polar angle plots of RAPID proton fluxes in the Mercator projection for the first energy channel (30-68 keV) during the relevant time interval. The fluxes were predominantly flowing northward and 

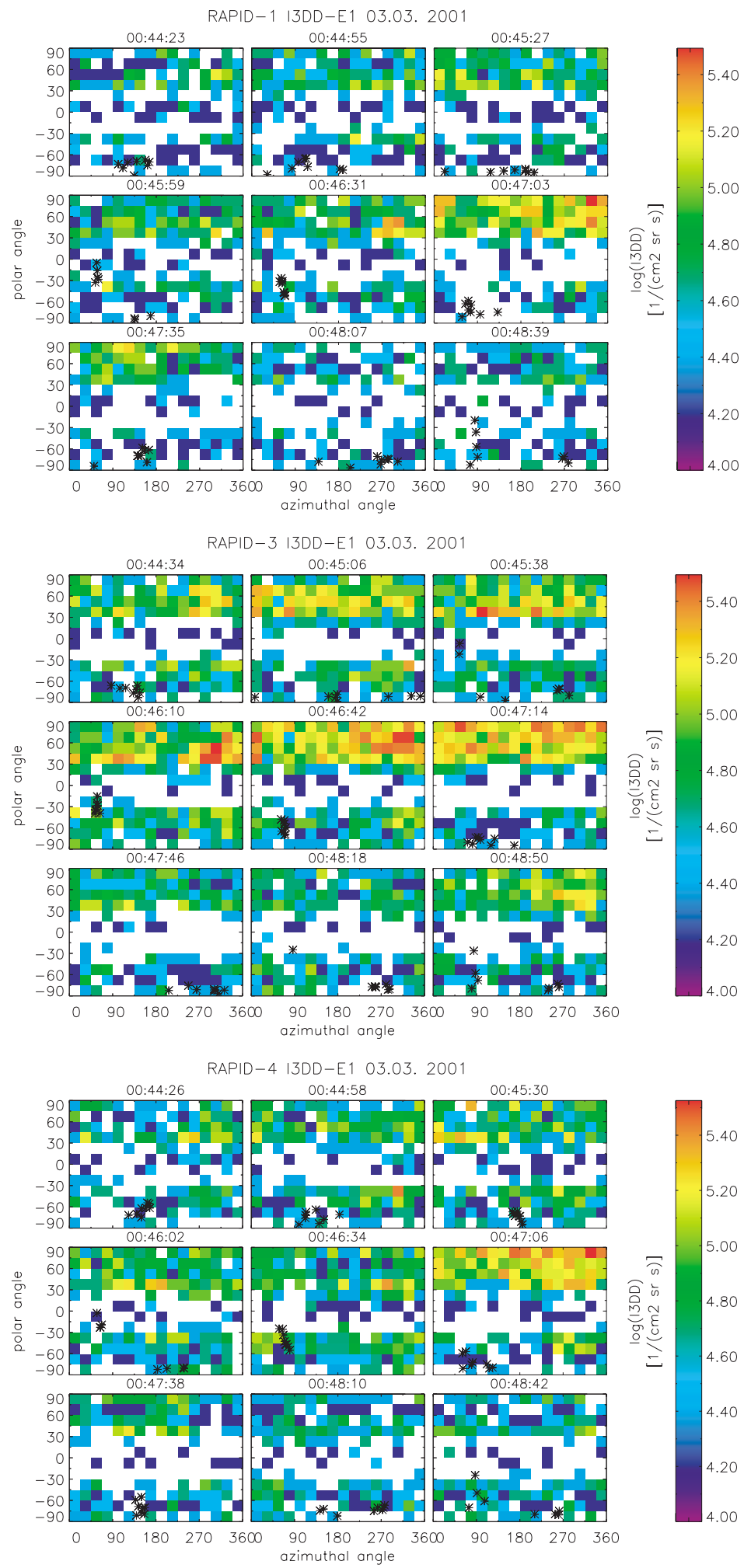

Fig. 10. Azimuthal-polar angle plots of proton ion fluxes, accumulated over 32 s, observed by RAPID in the first energy channel (30-68 keV) at the spacecraft $1,3,4$. The asterisks show the corresponding angles of the spin averaged (4s) magnetic field.

and in the morning direction, i.e. leaving the magnetosphere. The highest intensity was in the direction antiparallel to the magnetic field. Fluxes into other directions were also ob- served. In comparing the azimuthal-polar angle plots of the three spacecraft, one finds that spacecraft 3, located between spacecraft 1 and spacecraft 4 in the $Z_{\mathrm{GSE}}$ direction (see 
Fig. 9) observed longer and more intense fluxes. This can be interpreted as if spacecraft 1 and 4 observed the boundary of the ion flux structure while spacecraft 3 saw its center. Notice that the enhanced fluxes are observed only at the sunward half of the magnetic structure. The CNO particle fluxes, although at much lower count rates, exhibit the same variations as the protons.

\section{Summary and conclusions}

In December 2000 and early 2001, spacecraft traversed the dayside magnetopause repeatedly between dusk and the subsolar point. On 14 January 2001, the crossing occurred at 16:00LT. At the beginning of the pass, the spacecraft satellites came briefly in contact with trapped particles on the dawn side of the magnetosphere. Then the satellites apparently entered a region with open field lines which is not able to support energetic particles in large quantities. At 10:45 UT, spacecraft entered the trapped region at highlatitudes on the dusk side and left the magnetosphere at 15:10 UT. RAPID, with its unique capability of measuring the complete angular distribution of energetic particles, allows for the simultaneous measurement of local density gradients, as reflected in the anisotropies of $90^{\circ}$ particles and the remote sensing of changes in the distant field line topology, as reflected in the variations of loss cone properties. A detailed discussion of angle-angle plots shows considerable differences in the structure of the boundaries between the open and closed field lines on the nightside fraction of the pass and the magnetopause crossing. The crossing into the closed field lines in the nightside appears to be dominated by significant gradients of particle densitity. On the dayside magnetopause, on the other hand, the effect of density gradients is less obvious and the particle flux appears to be eroded by the growing size of the loss cones which, in the interpretation of the schematic in Fig. 9, results from reconnection at the magnetopause. These kind of detailed angular distributions obtained with high time resolution were not possible with the ISEE-1 scanning device.

On 3 March 2001, Cluster encountered just several FTEs outside the magnetosphere. The multi-spacecraft configuration allowed for the determination of the FTE properties. The FTE, which has been investigated in more detail, was moving primarily in an Earthward direction with a speed of $40 \mathrm{~km} / \mathrm{s}$. This is about two times slower than the motion of the surrounding plasma. We interpret this result observed by our multi-spacecraft investigation as being due to the anchoring of the FTE flux tube in the magnetosphere.

In the FTE, ions with energies larger than $30 \mathrm{keV}$ were leaving the magnetosphere in the northern/pre-noon direction, antiparallel to the magnetic field. We conclude that these regions were magnetically connected with the magnetic field of the Earth. The FTE signature is, indeed, created by the reconnection of magnetospheric and solar wind magnetic fields.

RAPID multi-spacecraft observations also allow for structure investigations. When spacecraft 3 was located in the central part of the connected flux tube, it saw more intense fluxes over a longer time. At the same time, spacecraft 1 and 4 observed the edge of the connected flux tube and saw lower flux intensities during a shorter time. The intense fluxes were only observed in the sunward part of the magnetic structure. Since this part was first seen by the spacecraft, this observation is similar to the plasma observation in the trailing part of FTEs made by Le et al. (1999).

Acknowledgements. The authors want to acknowledge the contribution to the Cluster RAPID sensor effort of W. Weiss, W. Güttler, K.-H. Otto, H. Wirbs at the Max-Planck-Institut für Aeronomie, D. Reading and S. Thomas at the Rutherford Appleton Laboratory and R. Cope at LANL. We appreciate the efforts of I. Güttler who supported us from the completion of various administative tasks up to testing activities. We thank I. Pardowitz and his team at the computer center of MPAE for implementing and maintaining the RAPID web site. We acknowledge the FGM team (PI: A. Balogh) for providing the magnetic field data. Work at MPAe was supported by DARA under Grant 50 OC 89097 . The work at RAL was funded by the UK Particle Physics and Astronomy Research Council.

Topical Editor M. Lester thanks C. Taquey and T. Rae for their help in evaluating this paper.

\section{References}

Daly, P. W., Saunders, M. A., Rijnbeek, R. P., Sckopke, N., and Russel, C.: The distribution of magnetic reconnection geometry in flux transfer events using energetic ion, plasma and magnetic field data, J. Geophys. Res., 89, 3843, 1984.

Escoubet, C. P., Schmidt, R., and Goldstein, M. L.: Cluster science and mission overview, Space Sci. Rev., 79, 11, 1997.

Le, G., Gosling, J. T., Russell, C. T., Elphic, R., and Newbury, J. A.: The magnetic and plasma structure of flux transfer events, J. Geophys. Res., 104, 233, 1999.

Papamastorakis, I.: Orientation, motion and other properties of flux transfer events structure on 4 September 1984, J. Geophys. Res., 94, 8852-8866, 1989.

Russell, C. T.: Reconnection at the Earth's amgentopause: Magnetic field observations and flux transfer events, Geophys. Monogr., (Ed) Hones, E. W., 30, 124, 1994.

Russell, C. T. and Elphic, R. C.: Initial ISEE magnetometer results: magnetopause observations, Space Sci. Rev., 22, 681, 1978.

Scholer, M., Ipavich, F. M., Gloeckler, G., Hovestadt, D., and Klecker, B.: Leakage of the magnetospheric ions into the magnetosheath along reconnected field lines at the dayside magnetopause, J. Geophys. Res., 86, 1299-1304, 1981.

Sonnerup, B. U. O. and Cahill, Jr., L. J.: Magnetopause strucure and attitude from explorer 12 observation, J. Geophys. Res., 73, 171, 1967.

Wilken, B., Axford. W. I., Daglis, I., et al.: RAPID: The Imaging Energetic Particle Spectrometer on Cluster, Space Science Review, 399-473, 1995. 